\title{
Vapour permeation for the recovery of organic solvents from waste air streams: separation capacities and process optimization
}

\author{
M. Leemann ${ }^{a} *$, G. Eigenberger ${ }^{a}$, H. Strathmann ${ }^{b}$ \\ "University of Stuttgart, Department of Chemical Engineering, Böblingerstr. 72, 70199 Stuttgart, Germany \\ ' University of Twente, Department of Chemical Technology, P.O. Box 217, 7500 AE Enschede. Netherlands
}

Accepted 3 April 1995

\begin{abstract}
Vapour permeation is a potentially suitable technology for the recovery of organic solvents from waste air streams. New solvent stable capillary membrane modules that are currently emerging on the market provide large membrane areas for an acceptable price and enhance the competitiveness of this process. Most membranes used in vapour permeation are silicone coated composites. Polydimethylsiloxane (PDMS) provides good separation capabilities and is highly permeable. The permeabilities for solvents and permanent gases show an inverse temperature dependence due to the different enthalpies of sorption and diffusion. Selectivities of silicone coated composite membranes are lower than that of pure PDMS but still high enough to enable a high degree of enrichment. The investigation of systems with two solvent components in air shows that selectivities and permeabilities are only slightly lower than in the case when only one solvent component is present. Coupling effects like preferential sorption are, therefore, not very strong.

The experimental results have been used as basis for an economical process optimization. Comparison with other waste air cleaning technologies shows that in the range of medium to high solvent concentrations and low to medium feed volume fluxes vapour permeation can be an economical alternative to the conventional processes.
\end{abstract}

Keywords: Waste air cleaning; Solvent recovery; Gas and vapor permeation; Diffusion; Solubility and partitioning

\section{Introduction}

In 1986 emissions of organic solvents into the air in Germany amounted to more than $950000 \mathrm{t} \mathrm{[1]}$. With regard to more strict environmental regulations there is an urgent need for efficient processes to reduce these emissions. On the other hand organic solvents are often valuable compounds and recovery of these substances is desirable in order to recycle them. Vapour permeation is a suitable recycling technology because during the recovery process the solvents are not subjected to

\footnotetext{
* Corresponding author.
}

high temperatures or changed in their chemical structure [2-8].

A schematic process flow sheet of a vapour recovery system utilizing semipermeable membranes is shown in Fig. 1.

Solvent contaminated air is fed to the membrane module and a solvent enriched air stream can be recovered as permeate. The retentate is a solvent depleted air. Typically the process is performed at nearly atmospheric pressure on the feed side and a vacuum of less than 200 mbar on the permeate side, since in this case no energy consuming compression of the feed and no installation of a feed side compressor are necessary. 


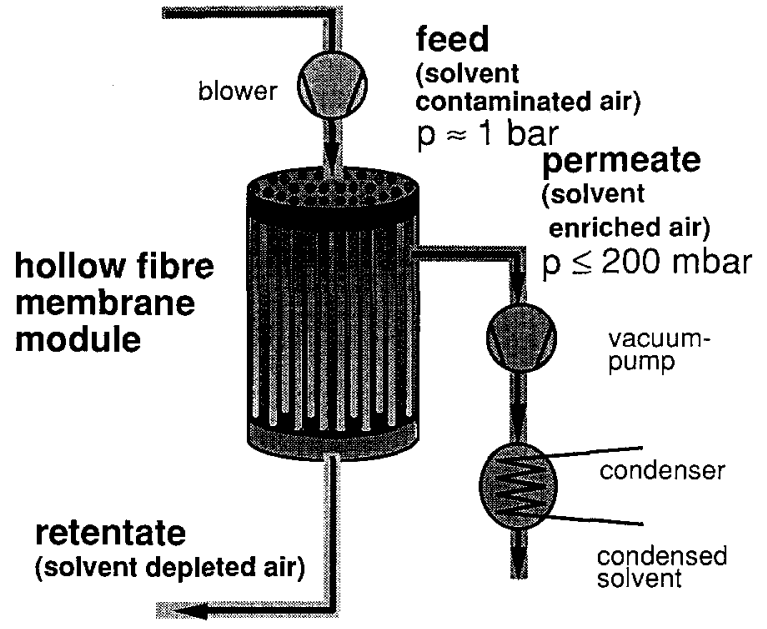

Fig. 1. Schematic diagram illustrating a solvent recovery system utilizing a hollow fibre membrane module.

Feed side compression is favourable only in case higher concentrated air streams have to be processed.

Until recently two main difficulties hindered a wider application of the process.

- The solvent stability of existing membranes was not sufficient or these membranes were only available in the form of flat sheets. In order to treat large low concentrated air streams it is necessary to install higher membrane areas. Therefore, it is mandatory to have a low cost membrane module with high packing density such as hollow fibre or capillary systems. These types of modules which provide large membrane areas at a reasonable price are currently emerging on the market.

- Furthermore, separation capacities, the optimal operating parameters and the area of economical application are still unknown for many applications.

\section{Experimental}

\subsection{Determination of membrane vapour permeability}

Most membranes used in vapour permeation are silicone coated composite membranes. Thin films (50$80 \mu \mathrm{m}$ ) from pure polydimethylsiloxane (PDMS; Wacker Chemie, Dehesive 920) were investigated with respect to their sorption, diffusion and permeation behaviour for organic solvents and permanent gases (Fig. 2).

A pure solvent vapour or a pure permanent gas is fed into a stirred membrane cell that is kept in an oil bath to guarantee isothermal conditions. Values for the permeability were obtained by two different methods:

According to the first method the mean permeability $\bar{P}$ in the pressure interval between $p^{\mathrm{F}}$ and $p^{\mathrm{P}}$ can be determined from the observed pressure drop in the feed side chamber:

$\bar{P}=\frac{-\frac{\mathrm{d} p^{\mathrm{F}}}{\mathrm{d} t} V^{\mathrm{F}} \delta^{\mathrm{M}}}{\left(p^{\mathrm{F}}-p^{\mathrm{P}}\right) A^{\mathrm{M}}} \cdot \frac{T^{0}}{p^{0} T}$

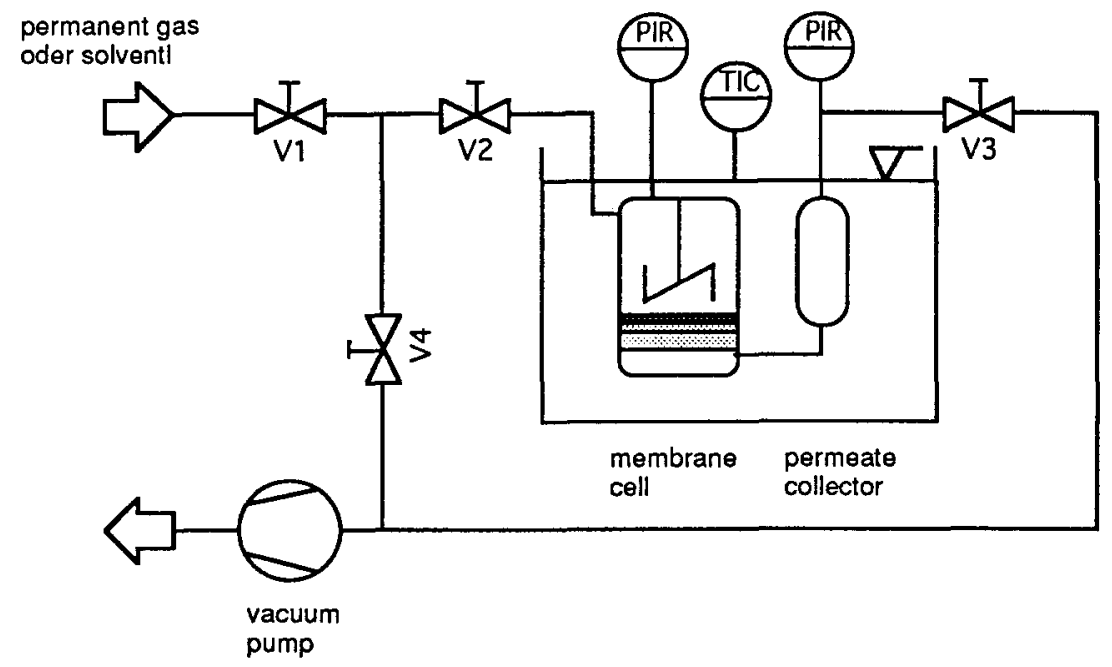

Fig. 2. Experimental set-up for the evaluation of permeabilities in one component systems. 
Table 1

Specification of investigated membrane modules

\begin{tabular}{ll}
\hline Number of fibres & $100-160$ \\
External diameter & $0.9-1.2 \mathrm{~mm}$ \\
Internal diameter & $0.7-1.0 \mathrm{~mm}$ \\
Membrane length & $250 \mathrm{~mm}$ \\
Membrane area & $700-950 \mathrm{~cm}^{2}$ \\
$\mathrm{O}_{2}$ permeability & $0.1-0.7 \mathrm{~m}^{3}(\mathrm{STP}) / \mathrm{m}^{2} \mathrm{~h} \mathrm{bar}$ \\
\hline
\end{tabular}

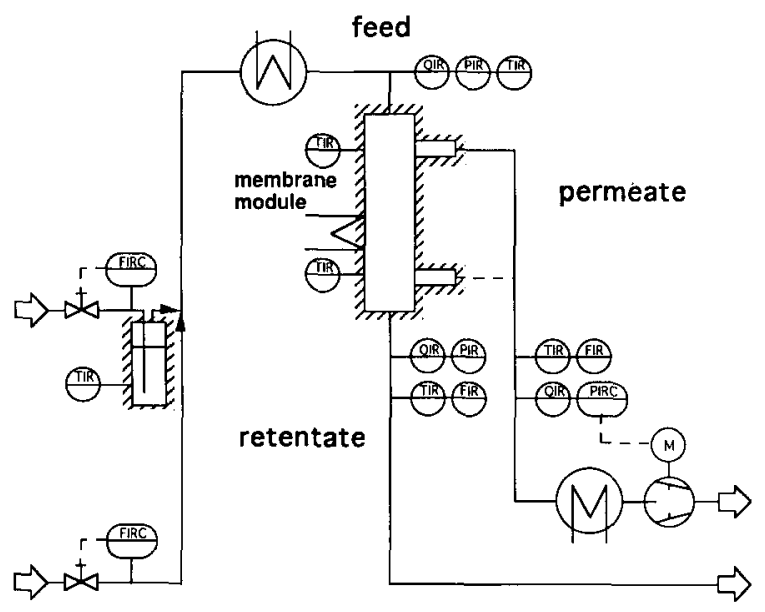

Fig. 3. Experimental set-up for module tests.

Table 2

Monitored operating parameters

\begin{tabular}{ll}
\hline Pressure & Feed side: 1 bar \\
& Permeate side: $10-200$ mbar \\
Temperature & $20-95^{\circ} \mathrm{C}$ \\
Systems & $\begin{array}{l}\text { One solvent component in air (toluene-air, ethyl } \\
\text { acetate-air) } \\
\text { Two solvent components in air (toluene-ethyl } \\
\text { acetate-air) }\end{array}$ \\
Concentration & $1000-10000 \mathrm{ppm}\left(\mathrm{ca} .4-40 \mathrm{~g} / \mathrm{m}^{3}(\mathrm{STP})\right.$ ) \\
\hline
\end{tabular}

Here $V^{\mathrm{F}}$ denotes the volume of the feed compartment, i.e. the stirred cell, $\delta^{\mathrm{M}}$ the film thickness, $A^{\mathrm{M}}$ the membrane area, $T$ the temperature, $p^{\mathrm{F}}$ and $p^{\mathrm{P}}$ the pressures on feed and permeate side. The index 0 specifies standard conditions. The permeability at a certain feed pressure $P\left(p^{\mathrm{F}}\right)$ can be calculated from:

$P\left(p^{\mathrm{F}}\right)=\vec{P}+\frac{\mathrm{d} \bar{P}}{\mathrm{~d} p^{\mathrm{F}}}\left(p^{\mathrm{F}}-p^{\mathrm{P}}\right)$

If the permeate side is continuously evacuated $\left(p^{\mathrm{P}}=0\right.$ mbar $)$ it follows
$P\left(p^{\mathrm{F}}\right)=\bar{P}+\frac{\mathrm{d} \bar{P}}{\mathrm{~d} p^{p}} p^{\mathrm{F}}$

The second method is based on the principle of pressure balance. After adjustment of a certain feed pressure the feed valve $V 2$ is closed and the permeate collector is also disconnected from the vacuum pump. Pressure decrease on the feed side and pressure increase on the permeate side should lead to the same mean permeability data. For an infinitely small pressure difference between feed and permeate the mean permeability approaches the permeability at a certain pressure

$P\left(p^{\mathrm{F}}\right)=\lim _{p^{\mathrm{P}} \rightarrow p^{\mathrm{F}}} \bar{P}$

Values for the permeability are determined with very little flux and after a certain stabilization time. This is especially useful for a fast permeating component in order to compensate for dynamic effects, such as adsorption, pressure drops in the sintered membrane support, etc., or if the permeability is strongly concentration dependent. The results are permeabilities at discrete pressures.

\subsection{Determination of membrane solvent sorption}

Sorption data were obtained by a gravimetric method. A small polymer sample was exposed to a solvent laden air stream of constant solvent concentration and the solvent uptake was determined by measuring the weight increase as a function of time. Equilibrium sorption was assumed when constant weight was reached.

\subsection{Investigation of capillary modules}

It was not clear whether permeabilities and ideal selectivities in pure silicone rubber derived from single component experiments could be used for the permeation of mixtures through composite membranes. Therefore, experimental studies with capillary membranes (PDMS on a substructure of polyether imide, PEI; the inside of the fibre is subjected with feed) have been performed. The membrane modules which consist of an exchangeable membrane cartridge installed in a stainless steel shell were supplied by GKSS, Forschungszentrum Geesthacht (Germany). 
The characteristics of the modules are listed in Table 1.

The experimental set-up and the analyzed operating parameters are shown in Fig. 3 and Table 2.

\section{Results and discussion}

\subsection{One component sorption, diffusion and permeation behaviour of PDMS}

The permeation behaviour of toluene and nitrogen in polydimethylsiloxane as a function of temperature and pressure is shown in Fig. 4. The data were obtained using the arrangement shown in Fig. 2.
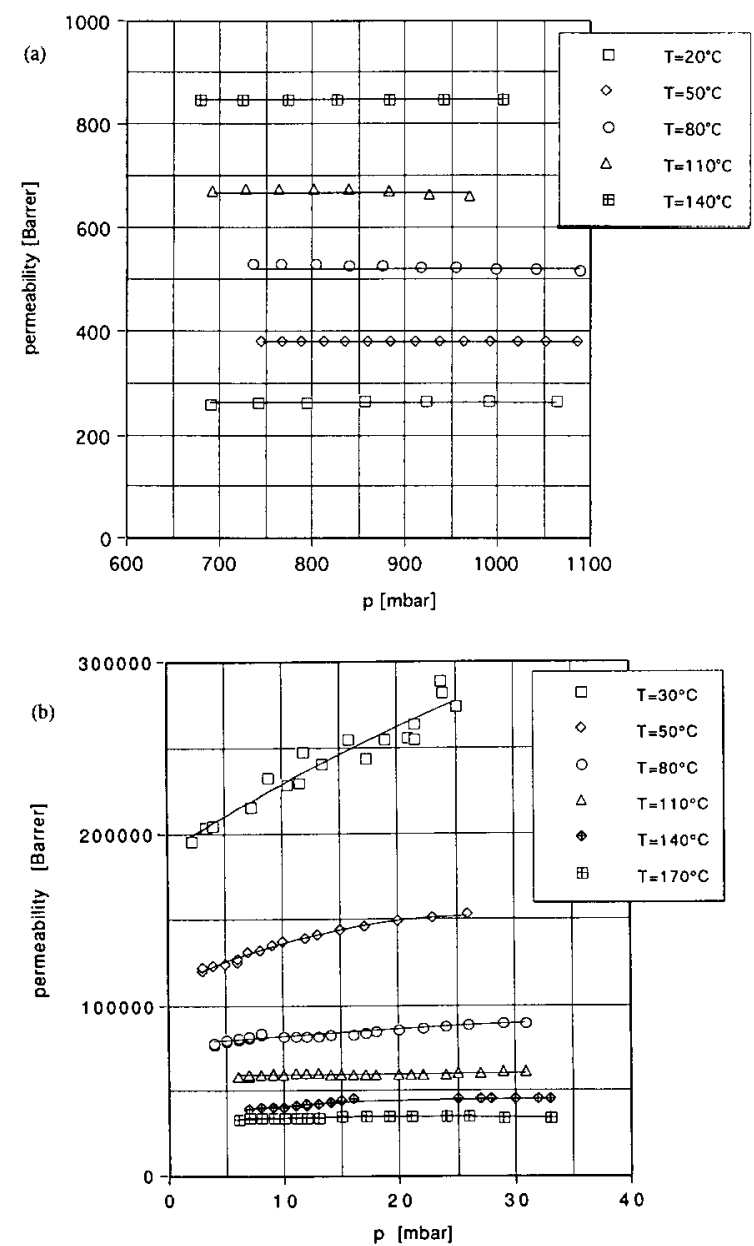

Fig. 4. Permeabilities of nitrogen (a) and toluene (b) in silicone rubber at different temperatures.
The permeability for toluene decreases with increasing temperature whereas it increases for nitrogen. Accordingly, the ideal toluene-nitrogen selectivity decreases from a value of 800 at a temperature of $30^{\circ} \mathrm{C}$ to 50 at $140^{\circ} \mathrm{C}$. At lower temperatures the permeability for the solvent increases rapidly with concentration, whereas for nitrogen the permeability is concentration independent at all temperatures. But even at higher temperatures silicone is much more permeable for toluene than for permanent gases such as oxygen or nitrogen.

The effect of temperature becomes even more clear if permeabilities are plotted in form of an Arrhenius diagram, as shown in Fig. 5.

The temperature dependence approximately follows the Arrhenius relationship. The different behaviour of solvents and permanent gases can be explained with the assumption of a solution-diffusion membrane transport mechanism and based on the temperature dependence of diffusivity $D$ and sorption coefficient $K$ according to an Arrhenius relationship:

$$
\begin{aligned}
& P=D \cdot K \\
& =D_{0} \exp \left(-E_{\mathrm{d}} / R T\right) \cdot K_{0} \exp \left(-\Delta h_{\mathrm{s}} / R T\right) \\
& =D_{0} \cdot K_{0} \exp \left(-\left(E_{\mathrm{d}}+\Delta h_{\mathrm{s}}\right) / R T\right)
\end{aligned}
$$

where $P$ is the permeability, $D$ the diffusion coefficient, $K$ the sorption or partition coefficient, $R$ the universal gas constant and $T$ the temperature. $E_{\mathrm{d}}$ refers to the activation energy for a diffusion step and $\Delta h_{\mathrm{s}}$ to the absorption enthalpy. $D_{0}$ and $K_{0}$ can be interpreted as diffusion or sorption coefficients at very high temperatures. For toluene diffusion is a slightly endothermal process $\left(E_{\mathrm{d}}>0\right)$, but absorption is strongly exothermic $\left(\Delta h_{\mathrm{s}} \ll 0\right)$. Therefore, $E_{\mathrm{d}}+\Delta h_{\mathrm{s}}$ is negative, i.e. the permeation is an exothermal process and the permeability must decrease with increasing temperature. The diffusion of nitrogen is also endothermal $\left(E_{\mathrm{d}}>0\right)$, but the absorption enthalpy is very low $\left(\Delta h_{\mathrm{s}} \approx 0\right)$. Therefore, $E_{\mathrm{d}}+\Delta h_{\mathrm{s}}$ is positive and thus the nitrogen permeability increases with increasing temperature.

The increasing permeability of solvents at lower temperature with increasing concentration can be understood from sorption measurements (Fig. 6).

For low to medium degrees of saturation the sorption is proportional to the applied vapour pressure, i.e. it can be described by Henry's law. Only at higher satu- 


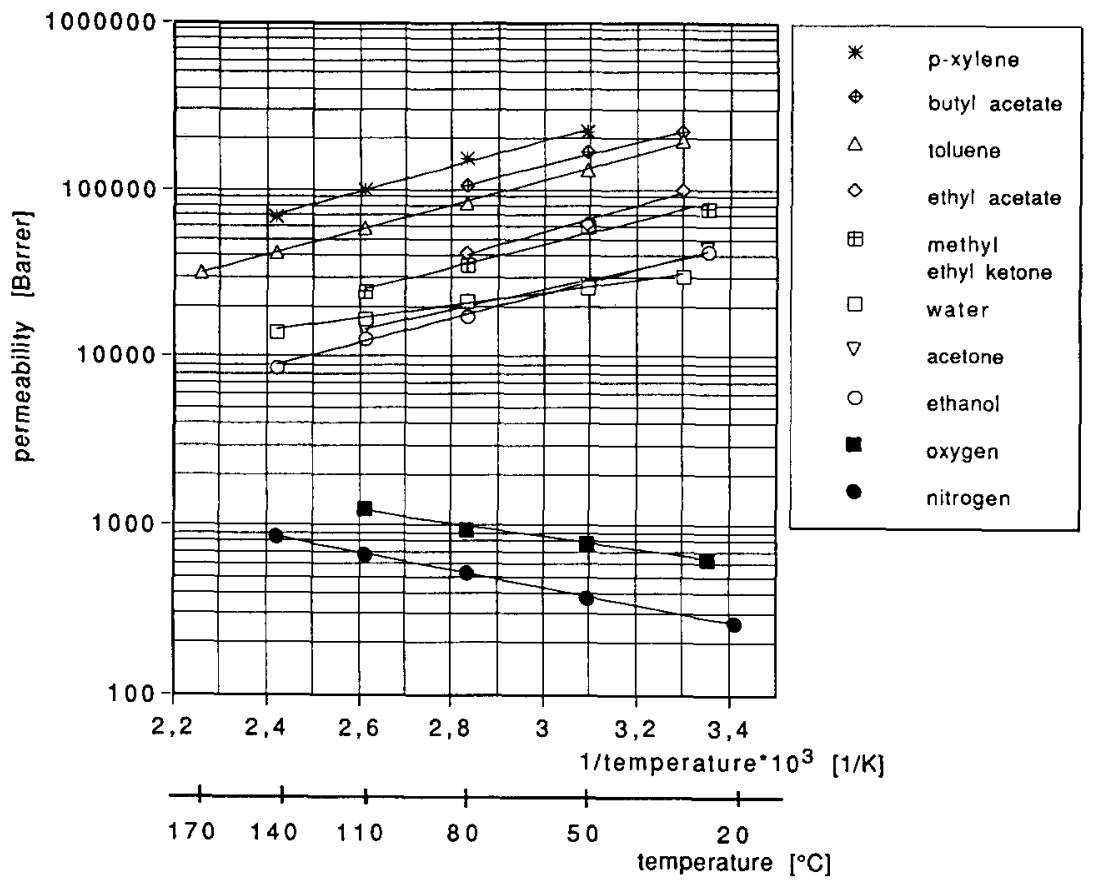

Fig. 5. Temperature dependence of permeabilities for various solvents and nitrogen (extrapolated to zero concentration).

ration the toluene sorption increases more than proportional with the vapour pressure.

Therefore, it can be derived from Eq. (4) that the increase in permeability with increasing partial solvent pressure as shown in Fig. 4 is due to an increase in diffusivity resulting from the high degree of polymer swelling.

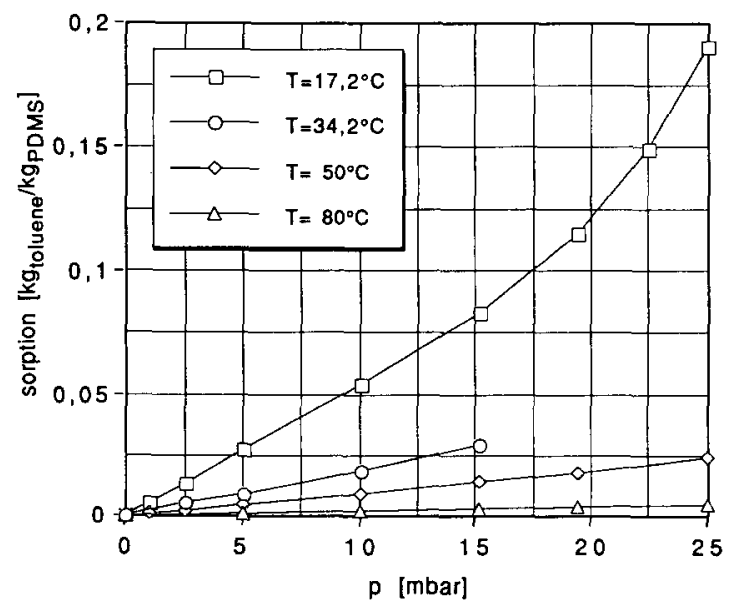

Fig. 6. Sorption isotherms of toluene in PDMS.

\subsection{Solvent recovery with capillary modules}

Fig. 7 shows enrichment factors and membrane fluxes for systems with one solvent component in air, i.e. toluene-air or ethyl acetate-air, and for a stage-cut of approximately zero, that means no solvent depletion occurred on the feed side. The investigations were performed with a capillary module specified in Table 1.

Due to the higher selectivity and permeability of PDMS for toluene, toluene can be higher enriched than ethyl acetate. The enrichment is always higher for lower temperatures which is apparent from the temperature dependence of the ideal selectivity as shown in Fig. 5. A good vacuum on the feed side leads with simple crossflow system design to satisfactory solvent purity. It seems surprising that although the permeability decreases with increasing temperature the highest solvent flux is achieved at elevated temperatures. This is due to the fact that the lower permeability at elevated temperatures is overcompensated by the increasing partial pressure difference between feed and permeate side due to the lower permeate concentration. The lower permeate concentration results from the lower selectivity at elevated temperatures. 

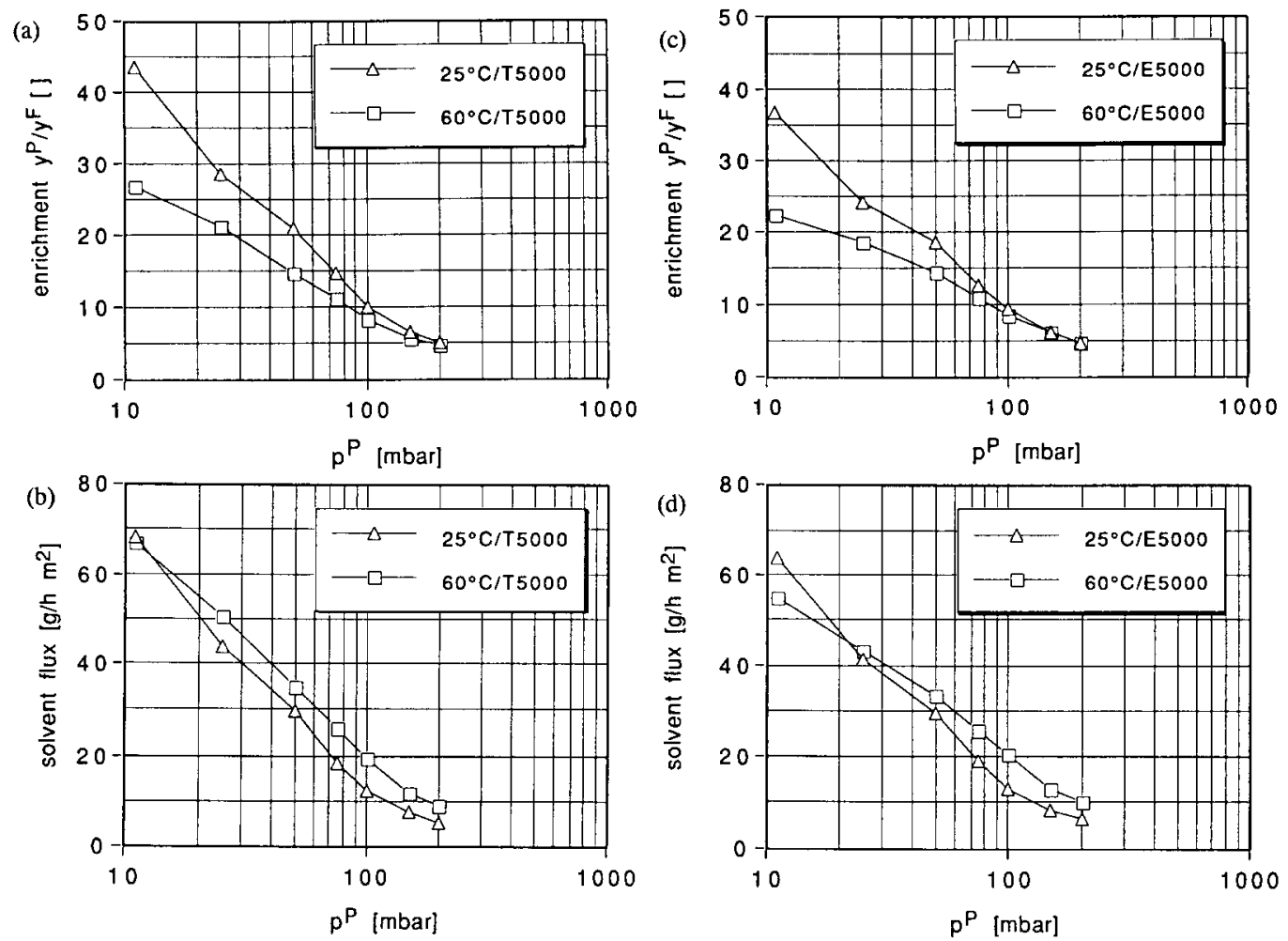

Fig. 7. Enrichment factor and solvent flux for toluene ( $\mathrm{a}$ and b) and for ethyl acetate (c and d) at temperatures of 25 and $60^{\circ} \mathrm{C}$ and a solvent concentration of $5000 \mathrm{ppm}$ in air; (stage-cut $\approx 0$ ).

For systems with two solvent components in air (toluene-ethyl acetate-air) solvent enrichment and solvent fluxes are very similar to the case when only one solvent component is present. Coupling effects like preferential sorption are therefore not very strong. This result contradicts at first sight the pressure or swelling dependent PDMS permeability but can be understood if the influence of the substructure is taken into account, since the resistance of the microporous substructure limits the solvent flux as shown later (Fig. 8) [9].

Typical results for the solvent enrichment on the permeate side, the solvent depletion on the feed side and the solvent fluxes for a stage-cut $\neq 0$ are shown in Fig. 9. Since the capillary membranes were relatively short $(25 \mathrm{~cm})$ and their diameter comparatively large $(1 \mathrm{~mm})$ only minor pressure drops occurred in the module. Therefore, the results are plotted over the ratio of feed standard volume flux and membrane area. Generally any degree of depletion can be achieved. Enrich- ment factors in the order of 20 are achievable for reasonable degrees of solvent depletion at permeate pressures of approximately 25 mbar.

\subsection{Optimal operating conditions and process costs}

The adjustment of operating parameters and installed membrane area may lead to any desired degree of depletion. The optimal values can be determined from a cost estimation and optimization. The calculations are based on experimental results with laboratory equipment and are upscaled to a feed flux of $1000 \mathrm{~m}^{3}$ (STP) / $\mathrm{h}$. The installed membrane area per module is $30 \mathrm{~m}^{2}$ and operation ( $3000 \mathrm{~h} /$ year) is performed in a single stage and in countercurrent flow.

As an example costs are calculated for a feed volume flux of $1000 \mathrm{~m}^{3}$ (STP) $/ \mathrm{h}$ with $5000 \mathrm{ppm}$ toluene in air, a process temperature of $T=25^{\circ} \mathrm{C}$, a permeate pressure of $30 \mathrm{mbar}$ in a solvent recovery rate of $70 \%$ and con- 

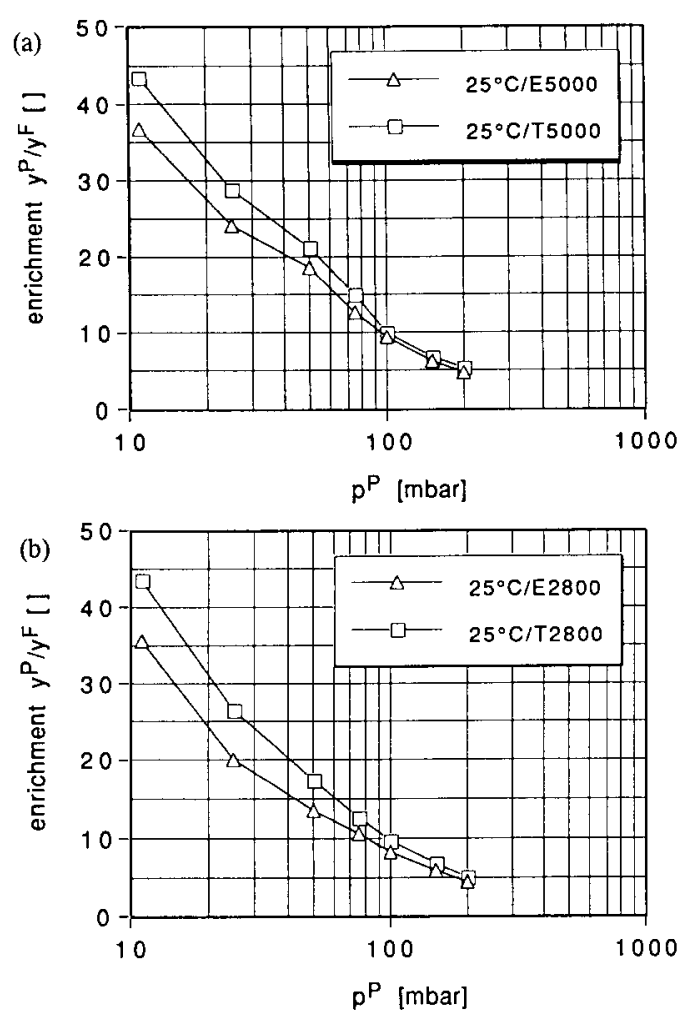

Fig. 8. Comparison of enrichment factors of toluene and ethyl acetate at $25^{\circ} \mathrm{C}$ for systems with one solvent component in air (a, $5000 \mathrm{ppm}$ toluene in air, $5000 \mathrm{ppm}$ ethyl acetate in air) and systems with two solvent components in air (b, 2800 ppm toluene and 2800 ppm ethyl acetate, air); stage-cut $\approx 0$.

densation of the solvent at $3^{\circ} \mathrm{C}$. The installed membrane area in this case is $179 \mathrm{~m}^{2}$ ( 6 modules in parallel), the permeate concentration $70000 \mathrm{ppm}$, which means a concentration factor of 13 . The permeate flux at 30 mbar is $1650 \mathrm{~m}^{3} / \mathrm{h}$ and $85 \%$ of the separated solvent vapour condenses in the liquid ring vacuum pump. Non-condensed permeate is recycled to the feed (Table $3)$.

Based on the results shown in Fig. 9, the annual cost varies as a function of the different operating parameters as shown in Fig. 10. A depletion of $70 \%$, for example, can be achieved either by a higher pressure on the permeate side and the installation of a large membrane area or by treating the feed at a low permeate pressure with a relatively small membrane area. A reduction of the permeate pressure leads to higher fluxes but requires the installation of a higher vacuum pump capacity. All iso-depletion lines in Fig. 10 show increasing costs with increasing permeate pressure. Therefore, the optimal permeate side operating pressure is in the range of 30 mbar, which is the lowest pressure that can be achieved with liquid ring pumps, if a feed concentration of 2000 to $10000 \mathrm{ppm}$ is treated and degrees of solvent depletion of $\leq 90 \%$ are anticipated. A further decrease of permeate side pressure leads to increasing costs because in this case much more expensive Roots pumps must be installed. The results obtained for the solvent toluene are also valid for other solvents with similar vapour pressures. The permeability of PDMS for other solvents is in most cases lower than the permeability for toluene. But since the resistance of the composite membrane can be interpreted as a serial arrangement of the resistances of the selective layer and the substructure and since the substructure resistance is in many cases dominating, the overall composite membrane selectivity and permeability for other solvents is only slightly lower than for toluene [9]. As a consequence optimal operation for a chosen depletion leads as a side effect to the highest degree of enrichment and the installation of smallest membrane areas. Optimal operation is also the most energy efficient operation as it is indicated in Fig. 10, because for constant depletion the energy consumption per $\mathrm{kg}$ of recovered solvent is approximately a contour line in Fig. 10.

The design of vapour recovery units operating in a single stage should be based on the following selection of operating parameters:

1. Choose a permeate pressure of $30 \mathrm{mbar}$.

2. Choose the desired degree of depletion or choose alternatively an enrichment factor.

3. Take from diagrams such as Fig. 9, the specific flux and calculate the necessary membrane area.

4. Choose liquid ring vacuum pumps and operate them at low temperature $\left(\approx 3^{\circ} \mathrm{C}\right)$.

In a so designed membrane solvent recovery plant vacuum pumps contribute $50 \%$ and membrane modules and measurement/control-units each approximately a quarter to the investment costs. Costs for energy affect a third and depreciation of membranes and the depreciation of other equipment each approximately a quarter of the annual costs. But it is worth noting that the membrane costs also influence capital costs and cost of maintenance, so that cheaper membranes significantly 

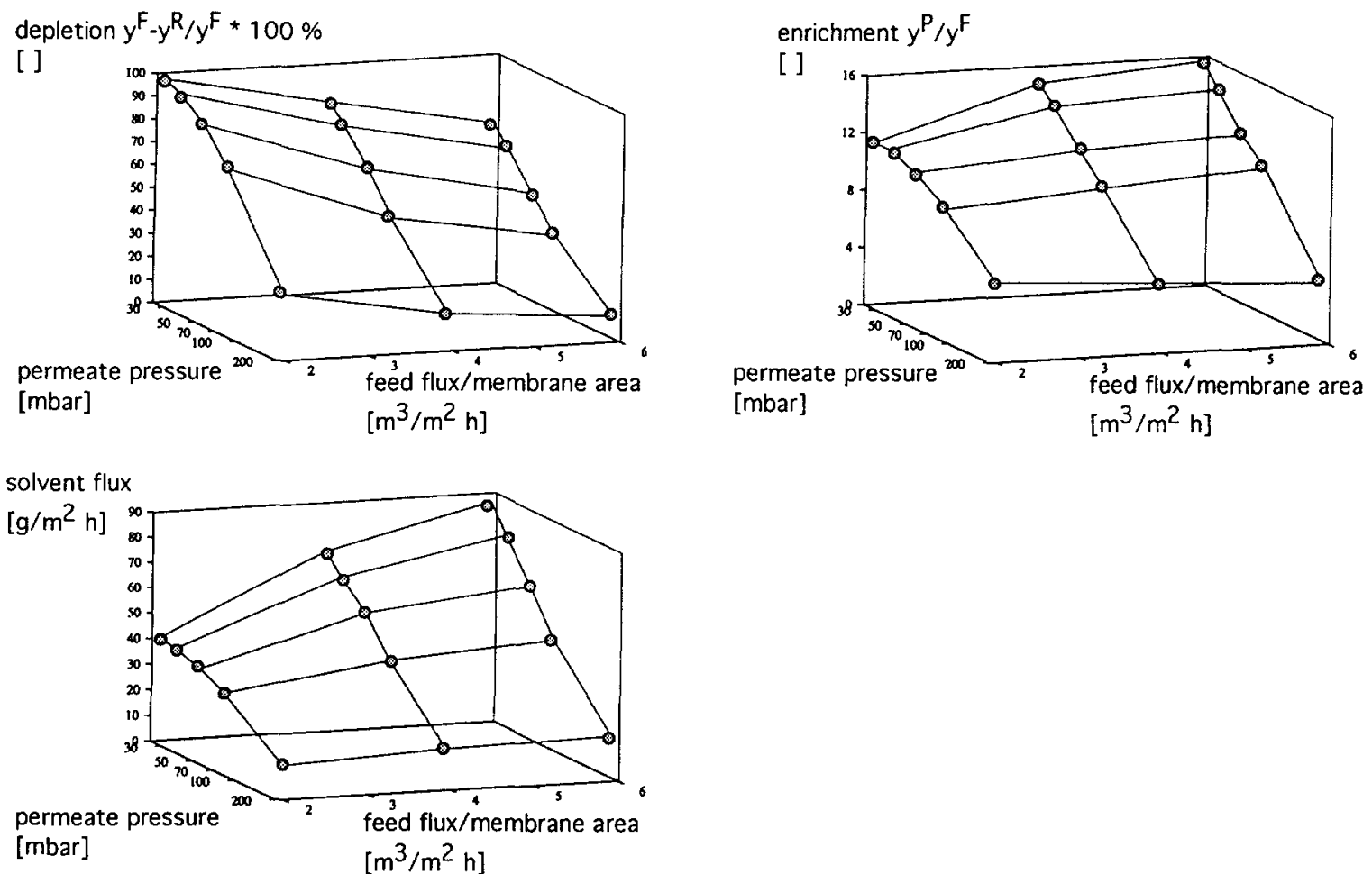

Fig. 9. Solvent depletion, enrichment and solvent fluxes for stage-cut $\neq 0$; feed: $5000 \mathrm{ppm}$ toluene in air, capillary module (fibre length $25 \mathrm{~cm}$, membrane area $A=785 \mathrm{~cm}^{2}$ ), operation in countercurrent flow at $T=25^{\circ} \mathrm{C}$
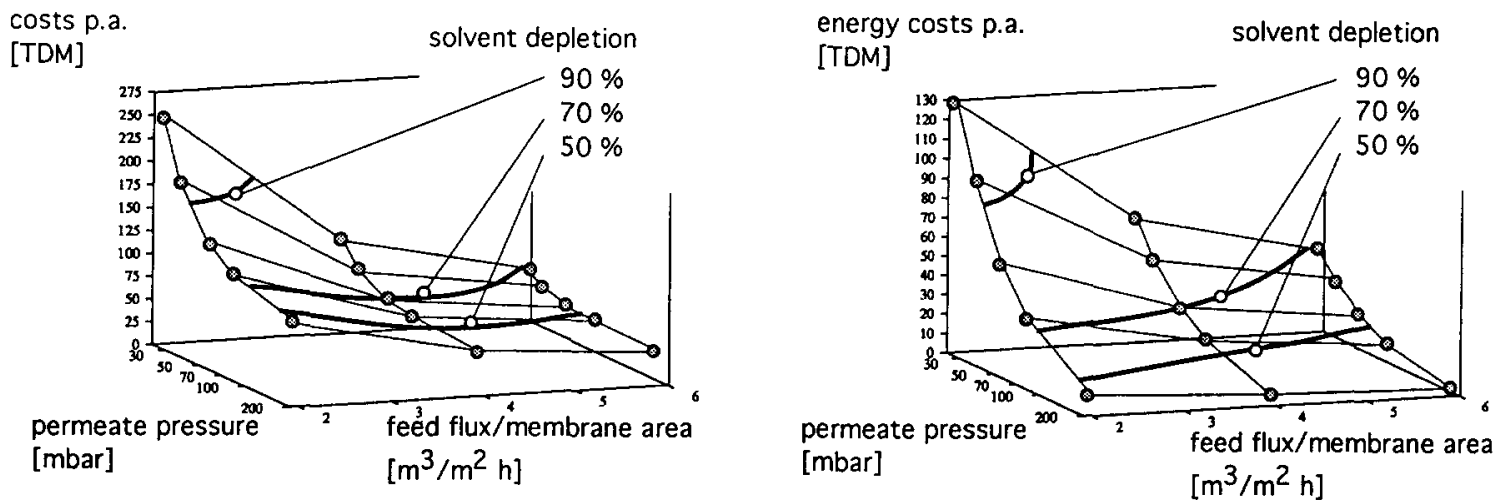

Fig. 10. Costs of a $1000 \mathrm{~m}^{3}$ (STP)/h capacity solvent recovery plant for different degrees of solvent depletion: (left) annual operating costs including profits from recovered toluene; (b) energy costs for different degrees of solvent depletion.

improve the competitiveness of the process. The same applies for profits from the recovered solvent (Fig. 11).

Comparison with costs of other air cleaning processes such as condensation, adsorption, absorption, catalytic and thermal combustion [10] shows that in the range of medium solvent concentrations (above several $\mathrm{g} / \mathrm{m}^{3}(\mathrm{STP})$ ) and low to medium feed volume fluxes (up to several thousand $\mathrm{m}^{3}(\mathrm{STP}) / \mathrm{h}$ ) vapour permeation can be an economical alternative to the conventional processes, providing: 
Table 3

Costs of a solvent recovery membrane system based on selective vapour permeation membrane, $n$ is the number of modules to be installed

Basis of calculation

Investment costs:

Piping, fittings, valves, flame barriers

Measurement and control instruments

Pressure transducer

Gas analyser (FID)

Multichannel recorder

control panel, etc.

Feed side blower

Vacuum pumps (liquid ring pumps, incl. explosion-

protection, on-site cooling available)

Membranes

Plant costs

Surcharges:

Projection, transport, assembling, start of operation

Expenditures to be paid by the client

Total investment:
Investment for $1000 \mathrm{~m}^{3} / \mathrm{h}$,

recovery $70 \%$

$\begin{array}{ll}1500 \mathrm{DM} \cdot n & 9000 \mathrm{DM} \\ 500 \mathrm{DM} \cdot n & 3000 \mathrm{DM} \\ 30000 \mathrm{DM} & 30000 \mathrm{DM} \\ 10000 \mathrm{DM} & 10000 \mathrm{DM} \\ 5000 \mathrm{DM} & 5000 \mathrm{DM} \\ 10000 \mathrm{DM} & 10000 \mathrm{DM} \\ 80 \mathrm{DM} / \mathrm{m}^{3} \mathrm{~h} \cdot V^{\text {permeate }} & 132000 \mathrm{DM} \\ \left(80000 \mathrm{DM} / 1000 \mathrm{~m}^{3} \mathrm{~h}\right) & \\ 250 \mathrm{DM} / \mathrm{m}^{2} & 44750 \mathrm{DM} \\ & 243750 \mathrm{DM} \\ & \\ 10 \% \text { of plant costs } & 24375 \mathrm{DM} \\ 4 \% \text { of plant costs } & 9750 \mathrm{DM} \\ & 277875 \mathrm{DM}\end{array}$

Basis of calculation

Annual cost for $1000 \mathrm{~m}^{3} / \mathrm{h}$, ecovery $70 \%$

Annual operating costs: (p.a. = per annum)

Fixed costs

Depreciation of equipment

Depreciation of membranes

$10 \%$ of investment without membranes p.a.

$33 \%$ of membranes p.a.

22690 DM p.a.

$10 \%$ of half investment p.a.

17000 DM p.a.

13890 DM p.a.

$2.5 \%$ of investment p.a.

6950 DM p.a.

Variable costs:

Electric energy

$0.15 \mathrm{DM} / \mathrm{kW} \mathrm{h}$

$10 \mathrm{~kW} \quad 4500$ DM p.a.

-feed side blower

$40 \mathrm{~kW} /\left(1000 \mathrm{~m}^{3} / \mathrm{h}\right.$ permeate volume flux $)$

$1 \%$ of investment $/ 1000 \mathrm{~h}$ operating time

29710 DM p.a.

8340 DM p.a.

Maintenance

Without profits from recovered solvent:

Annual costs:

$103080 \mathrm{DM}$ p.a

$0.034 \mathrm{DM} / \mathrm{m}^{3}$

$2.39 \mathrm{DM} / \mathrm{kg}$

Solvent recovery costs:

43050 DM p.a.

$1 \mathrm{DM} / \mathrm{kg}$ recovered solvent

60030 DM p.a.

$0.020 \mathrm{DM} / \mathrm{m}^{3}$

$1.39 \mathrm{DM} / \mathrm{kg}$ 

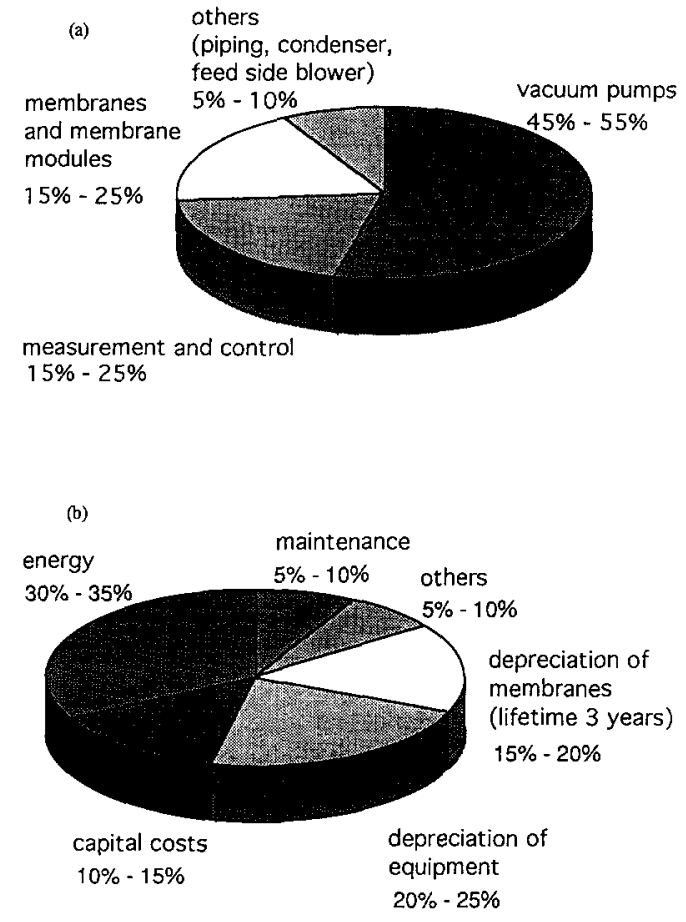

Fig. 11. (a) Contribution of different system components to the investment cost. (b) Contribution of different factors to annual cost in the case study.

1. Direct recycling of the solvents is possible (profits from recovered solvent).

2. Incomplete solvent depletion $(\leq 70 \%)$ can be tolerated.

\section{Acknowledgements}

The work described in this paper was supported by Kraftanlagen AG, Heidelberg, Germany and the Bundesministerium für Forschung und Technologie (BMFT).

\section{References}

[1] Umweltbundesamt (Ed.), Daten zur Umwelt 1988/89, Erich Schmidt Verlag, Berlin, 1989.

[2] R.W. Baker, N. Yoshioka, J. Mohr and A.J. Khan, Separation of organic vapour from air, J. Membrane Sci., 31 (1987) 259.

[3] M. Katoh, N. Inoue, T. Bitoo, K. Hashimoto, H. Tsuneizumi and N. Furuno, Hydrocarbon vapour recovery with membrane technology, NKK Techn. Rev., 56 (1989) 67.

[4] H. Paul, C. Philipsen, F.J. Gerner and H. Strathmann, Removal of organic vapours from air by selective membrane permeation, J. Membrane Sci., 32 ( 1988) 363.

[5] R.D. Behling, K. Ohlrogge, K.-V. Peinemann and E. Kyburz, The separation of hydrocarbons from waste air streams, AIChE Symp. Ser., 248 (1986) 82.

[6] H. Strathmann, Rückgewinnung von Lösemittel aus Abluft über Membranverfahren, in D. Ondratschek and K. Ortlieb (Eds.) Taschenbuch für Lackierbetriebe 1989, Curt R. Vincentz Verlag, Hannover, 1989.

[7] R.J. Lahiere, M.W. Hellums, J.G. Wijams and J. Kaschemekat, Membrane vapour separation. Recovery of vinyl chloride monomer from PVC reactor vents, Ind. Eng. Chem. Res., 32 (1993) 2236-41.

[8] I. Blume, P.J.F. Schwering, M.H.V. Mulder and C.A. Smolders, Vapour sorption and permeation properties of polydimethylsiloxane films, J. Membrane Sci., 61 (1991) 85 .

[9] M. Leemann, G. Eigenberger and H. Strathmann, Vapour permeation for the recovery of organic solvents from waste air streams: analysis of mass transfer in composite membranes, $\mathrm{J}$. Membrane Sci., submitted.

[10] Verein Deutscher Ingenieure (Ed.), Stofftrennverfahren in der Umwelttechnik, Conference Preprint, Köln, 1990. 